\title{
Primary nephrotic syndrome complicated by chylothorax: a case report
}

\author{
Yongwei $\mathrm{Xu}^{1} \wedge$, Jinpeng $\mathrm{Shi}^{2} \wedge$, Shuchang $\mathrm{Xu}^{1 *} \wedge$, Chunli $\mathrm{Cui}^{3 *} \wedge$ \\ ${ }^{1}$ Department of Gastroenterology, Tongji Hospital, Tongji University School of Medicine, Shanghai, China; ${ }^{2}$ Department of Medical Oncology, \\ Shanghai Pulmonary Hospital \& Thoracic Cancer Institute, Tongji University School of Medicine, Shanghai, China; ${ }^{3}$ Department of Nephrology, \\ Tongji Hospital, Tongji University School of Medicine, Shanghai, China \\ *These authors contributed equally to this work. \\ Correspondence to: Chunli Cui. Chief Doctor, Professor, Department of Nephrology, Tongji Hospital, Tongji University School of Medicine, No. \\ 389 Xincun Road, Putuo District, Shanghai 200065, China. Email: cuichunlispring@sina.com; Shuchang Xu, PhD, MD. Chief Doctor, Professor, \\ Department of Gastroenterology, Tongji Hospital, Tongji University School of Medicine, No. 389 Xincun Road, Putuo District, Shanghai 200065, \\ China. Email: xschang@163.com.
}

\begin{abstract}
Chylothorax is an uncommon and serious clinical condition, typically induced by trauma, either postsurgical or accidental injury, but the mechanism of chylothorax caused by nephrotic syndrome is still unclear. Here, we report a case of primary nephrotic syndrome with membranous nephropathy (MN) in a 66-year-old man who presented with severe chylothorax. The chylothorax was managed by intercostal chest tube drainage, subcutaneous injection of enoxaparin, and treatment with anti-inflammatory agents and diuretics. After treatment, the patient's pleural effusion decreased, and the chyle gradually became clear. We discuss the causes of MN with chylothorax. We considered that the hypoproteinemia changed the permeability of mucous membranes and lymphatic vessels, leading to leakage of chylous particles and chylous pleural effusion formation. Chylothorax may also have been caused by severe tissue edema, edema of the lymphatic walls, and increased pressure, resulting in increased permeability or rupture of the lymphatic wall, and leakage of chylous fluid into the thoracic cavity. Because of its rarity, we hope this case report will improve clinicians' understanding of MN complications in primary nephrotic syndrome and provide suitable treatment options for future clinical reference.
\end{abstract}

Keywords: Chyle; chylothorax; membranous nephropathy (MN); primary nephrotic syndrome; case report

Submitted Jul 08, 2021. Accepted for publication Sep 29, 2021.

doi: 10.21037/apm-21-1872

View this article at: https://dx.doi.org/10.21037/apm-21-1872

\section{Introduction}

Chyle is a milky fluid containing fat droplets and drains from the lacteals of the small intestine into the lymphatic system during digestion (1). The lymphatic channels coalesce to form the thoracic duct, collecting and transporting the chyle, ultimately draining into the left subclavian vein (2). Chylothorax can develop due to trauma, malignancy, tuberculosis, heart failure, lymphangiomatosis, or sometimes unidentifiable causes (3). Primary nephrotic syndrome is recognized as a rare cause of chylothorax, but the mechanism of chylothorax caused by primary nephrotic syndrome is unclear. We report a case of primary nephrotic syndrome in a male patient who developed chylothorax, highlighting how identifying the source of chylothorax has important clinical implications, avoiding unnecessary surgical exploration of the chest for diagnosis/or therapy.

^ ORCID: Yongwei Xu, 0000-0002-7416-4281; Jinpeng Shi, 0000-0001-5140-3238; Shuchang Xu, 0000-0002-4841-462x; Chunli Cui, 0000-00032033-6524. 


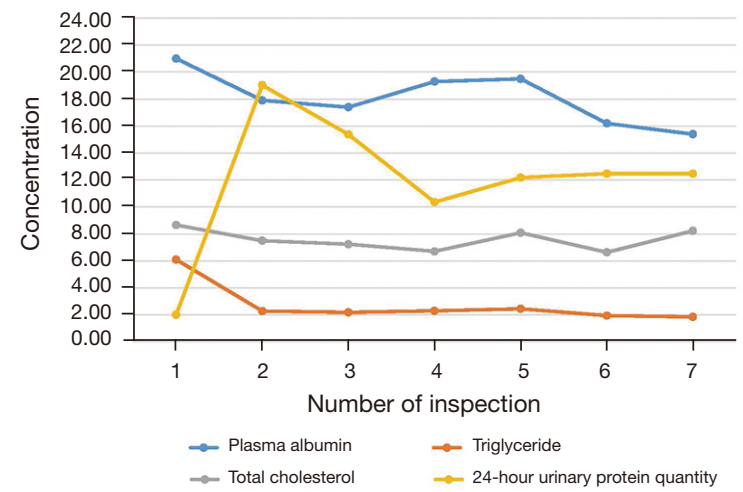

Figure 1 The results of each examination after the patient was admitted. Plasma albumin (reference range, 40-55 g/L), triglyceride (reference range, 0-1.7 mmol/L), total cholesterol (reference range, 3.1-5.2 $\mathrm{mol} / \mathrm{L}$ ), 24-hour urinary protein quantity (reference range, $0.028-0.14 \mathrm{~g} / 24 \mathrm{~h})$.

This report supplements the clinical data regarding typical primary nephrotic syndrome, complicated with chylothorax, assisting clinicians in improving the diagnosis, treatment, and prognosis of this rare condition. We present the case in accordance with the CARE reporting checklist (available at https://apm.amegroups.com/article/view/10.21037/apm$21-1872 / \mathrm{rc})$.

\section{Case presentation}

A 66-year-old man was admitted to the hospital 5 years ago for elevated blood pressure, massively foamy urine, and edema of the lower extremities. He did not have a history of heart disease, diabetes, cerebrovascular disease, mental illness, or any infectious diseases. Over the next 5 years, the patient had repeated hospital admissions for treatment. Before the latest admission, his blood pressure was serially monitored, and the highest recorded blood pressure was $185 / 105 \mathrm{mmHg}$. However, the patient was not optimally attended to or treated regularly, who took antihypertensive drugs intermittently to control his blood pressure. Multiple examinations revealed massive proteinuria, hypoproteinemia, hyperlipidemia, and obvious edema of both lower extremities. Repeated treatment such as diuretics (furosemide and spironolactone), commonly used antihypertensive drugs, and atorvastatin to lower blood lipids was administered. Laboratory tests revealed that indicators were much higher than normal values (Figure 1). Briefly, the results of post-admission blood tests were: albumin $16.2 \mathrm{~g} / \mathrm{L}$ (normal range, $40-55 \mathrm{~g} / \mathrm{L}$ ); creatinine
$163 \mu \mathrm{mol} / \mathrm{L}$ (normal range, $66-115 \mu \mathrm{mol} / \mathrm{L}$ ); total cholesterol $6.64 \mu \mathrm{mol} / \mathrm{L}$ (normal range, 3.1-5.2 $\mu \mathrm{mol} / \mathrm{L}$ ); triglycerides $1.95 \mathrm{mmol} / \mathrm{L}$ (normal range, $0-1.7 \mathrm{mmol} / \mathrm{L}$ ); leukocyte count $8.67 \times 10^{9} / \mathrm{L}$ (normal range, $3.5-9.5 \times 10^{9} / \mathrm{L}$ ); C-reactive protein $3.03 \mathrm{mg} / \mathrm{L}$ (normal range, $<10 \mathrm{mg} / \mathrm{L}$ ); and procalcitonin $0.089 \mathrm{ng} / \mathrm{mL}$ (normal range, $<0.05 \mathrm{ng} / \mathrm{mL}$ ).

At his first hospital admission 5 years ago, a renal puncture to confirm the pathological type of nephrotic syndrome showed membranous nephropathy (MN) (Figure 2). The patient was also tested for phospholipase A2 receptor (PLA2R), and the significant increase confirmed the diagnosis of $\mathrm{MN}$ (32 RU/mL, reference range, $<20 \mathrm{RU} / \mathrm{mL}$ ). Due to the increased edema of the lower extremities, another PLA2R test was performed after re-admission hospitalization. The concentration had increased to $143 \mathrm{RU} / \mathrm{mL}$, and rituximab was prescribed for treatment, but he declined continuous treatment due to the patient's consideration of cost.

A week ago, in May 2020, the patient experienced chest pain and was re-admitted to the hospital. Physical examination showed weakened breathing movement on the right side of the chest, with no pleural friction on auscultation and decreased breath sounds on both sides of the chest, but especially on the right side. No obvious abnormal signs were found during the physical examination. Chest computed tomography (CT) revealed a large pleural effusion (Figure $3 A, 3 B$ ), but it is difficult to distinguish the nature of the pleural effusion by CT alone. Therefore, laboratory examination of the fluid is very important (4).

Pleural drainage was performed after 1 week of active treatment to relieve the chest distress and discomfort. The patient's symptoms were obviously relieved, and a repeat chest CT showed that the pleural effusion was significantly reduced (Figure 3C,3D). The drained effusion was a milky white liquid (Figure 4). Further examination and analysis ruled out tumors, tuberculosis, and other infectious diseases, and biochemical analysis of the composition of the pleural effusion revealed a chylous pleural effusion (Table 1). Based on these results, a final diagnosis of primary nephrotic syndrome with chylothorax was made. We retested the level of PLA2R, and although it decreased to $97 \mathrm{RU} / \mathrm{mL}$, it was still higher than the normal value.

The patient was followed up after discharge, and reported neither chest tightness nor asthma, and other symptoms also improved significantly. The patient was advised to take the diuretic and other medications regularly to prevent symptoms causing discomfort, and there has been no recurrence of pleural effusion during follow-up. 

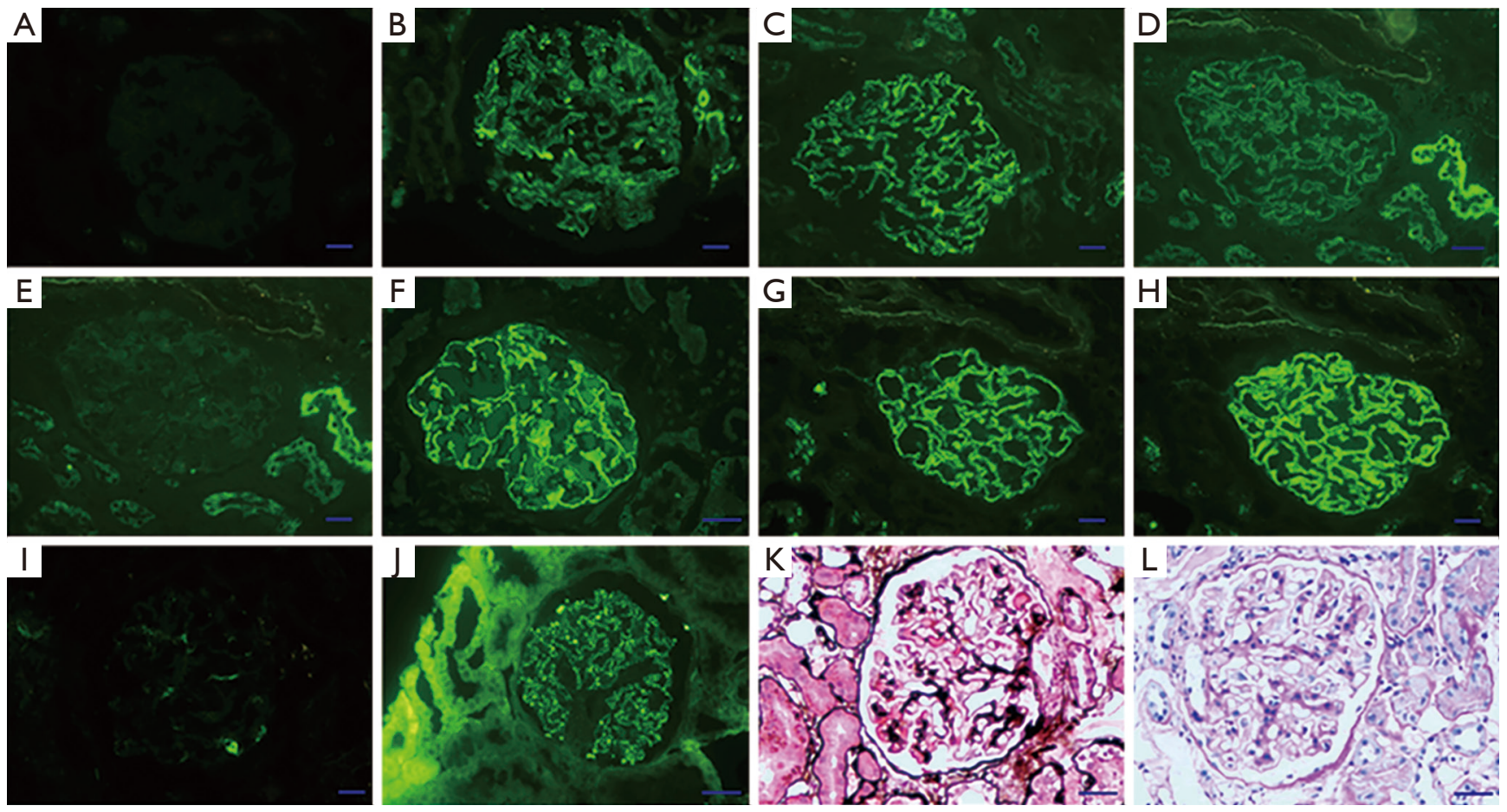

Figure 2 Results of immunofluorescence and light microscopic examination of glomeruli. (A) Shows glomerular IgA, (B) C3, (C) IgG1, (D) IgG2, (E) IgG3, (F) IgG4, (G) Kappa, (H) Lambda, (I) IgM and (J) PLA2R (100×). (K) and (L) (40×), under light microscope, the glomerular capillary basement membrane thickened, the basement membrane showed nail-like protuberance, and the foot process cells fused.

All procedures performed in this study were in accordance with the ethical standards of the institutional and/or national research committee(s) and with the Helsinki Declaration (as revised in 2013). The patient gave written informed consent for publication of this case report and accompanying images. A copy of the written consent is available for review by the editorial office of this journal.

\section{Discussion}

Disease progression and prognosis in adult nephrotic syndrome differ among patients, but a primary nephrotic syndrome with massive chylothorax has been rarely reported. Chylothorax results from chyle leakage from the lymphatic system into the pleural space, usually secondary to disruption of thoracic lymphatics. The intestine absorbs long-chain triglycerides in the diet, which are transformed into chylomicrons and very-low-density lipoproteins collected by the lymphatic vessels, then the thoracic duct, and transported as chyle that ultimately drains into the left subclavian vein.

The thoracic duct starts from the cisterna chyli, proceeds cranially along the right side of the spine, crosses the spine at the level of the fifth thoracic vertebra, and continues cranially along the left side of the esophagus. Therefore, chylothorax caused by thoracic duct injury or obstruction above the fifth thoracic vertebra mostly occurs on the left side of the chest, and if below the fifth thoracic vertebra, it usually occurs on the right side. Any injury to the duct as it courses through the thoracic cavity can lead to chylous effusion.

Circulating serum autoantibodies against the M-type PLA2R is a key biomarker in diagnosing and monitoring primary MN. PLA2R-associated MN most likely develops through factors such as genetic susceptibility, loss of tolerance, and alterations in antigen expression, and there is a role for environmental factors such as air pollution, smoking, and infections. Monitoring the concentration of serum anti-PLA2R antibody in patients with primary $\mathrm{MN}$ can evaluate the activity and recovery of the disease, and assess the severity of the condition before and after treatment, which can guide treatment and selection of drugs.

Because chylothorax is usually due to thoracic trauma or tumors, assessment of thoracic tomography scans 

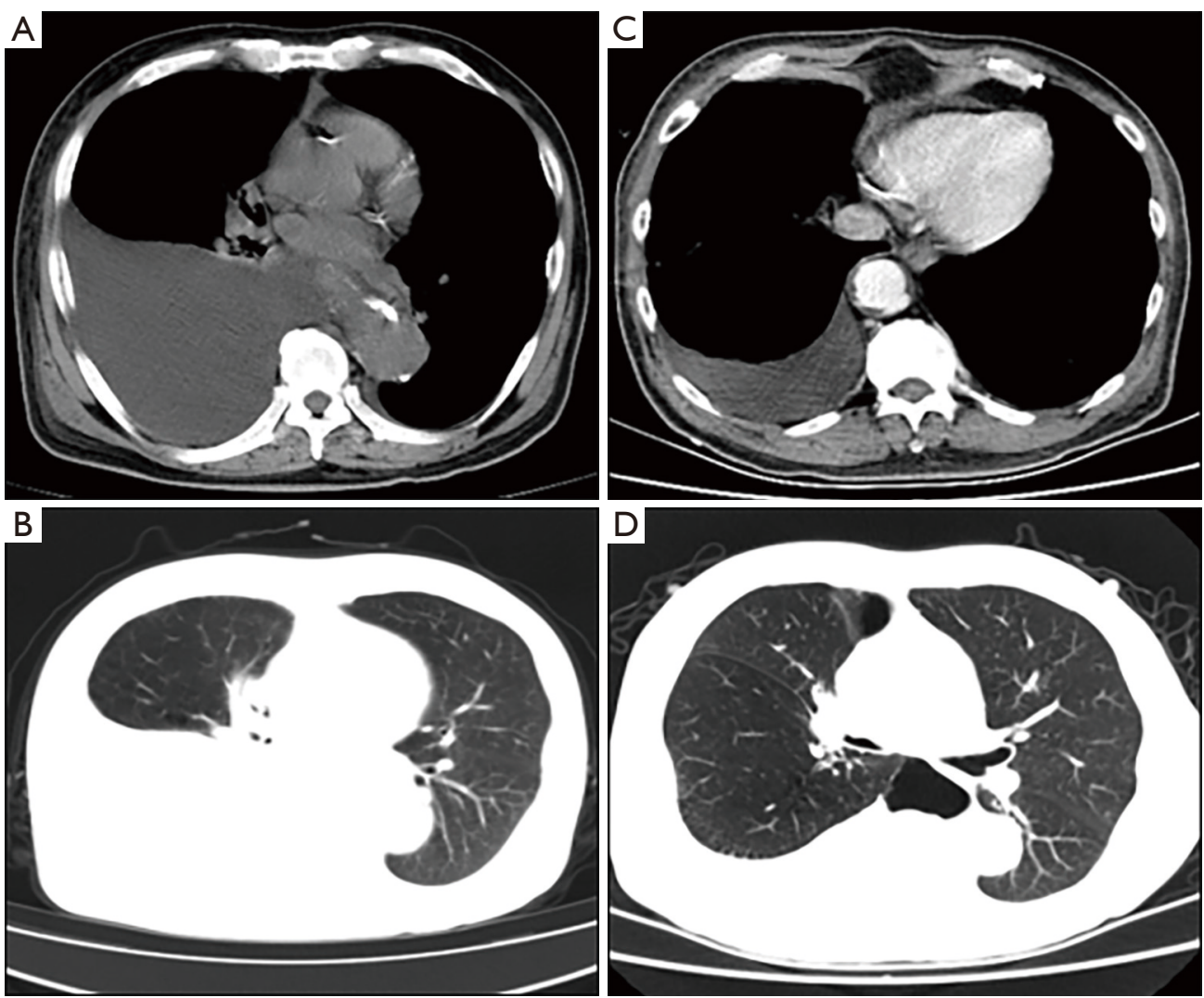

Figure 3 Images of computed tomography before (A,B) and after therapy (C,D). (A) Before treatment, there is a large amount of pleural effusion in the pleural cavity; (B) the right lung is compressed and reduced by pleural effusion. (C) After treatment, pleural effusion was significantly reduced and (D) the right lung tissue of was dilated.

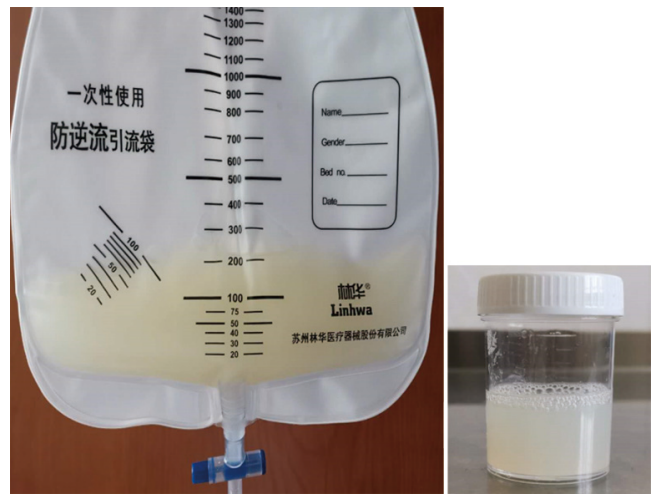

Figure 4 Cloudy milky pleural effusion immediately after thoracentesis.

and fluid evaluation are critical for eliminating possible malignancy. Apart from those main causes, filariasis, lymphangioleiomyomatosis, cirrhosis, pericarditis, sarcoidosis, tuberculosis, and the post-radiotherapy stage of tumor treatment may also induce chylothorax (5). In the present case, these causes were ruled out after examination of the pleural effusion. Therefore, the chylothorax was considered caused by the $\mathrm{MN}$ form of primary nephrotic syndrome.

Primary nephrotic syndrome is a common clinical renal disease caused by severe hypoproteinemia, and the incidence of serous effusion is high. The pleural effusion in nephrotic syndrome is usually a clear transudate. Effusion due to chylothorax is rarely reported (6). If the pleural effusion is chylous, it is necessary to distinguish between true chylous and pseudochylous effusion.

Pseudochylothorax also has a milky, turbid fluid due to elevated levels of cholesterol or phosphatidylcholine globulin complex, which often occurs after chronic infections. Pseudochylothorax can occur with tuberculosis, rheumatoid arthritis, and diabetes. 
Table 1 Pleural effusion test results

\begin{tabular}{|c|c|c|}
\hline Laboratory tests & Result & $\begin{array}{c}\text { Reference } \\
\text { ranges }\end{array}$ \\
\hline $\mathrm{pH}$ & 7.5 & $7.35-7.45$ \\
\hline Colour & White & Colorless \\
\hline Transparency & Turbid & Transparent \\
\hline Clot & - & - \\
\hline Rivalta & ++ & - \\
\hline Chylous test & + & - \\
\hline Total cholesterol, TC, mmol/L & 0.26 & $3.1-5.2$ \\
\hline Triglyceride, TG, mmol/L & 1.88 & $0-1.7$ \\
\hline Nucleated cell count, $\times 10^{6} / \mathrm{L}$ & 38 & $0-15$ \\
\hline Hydrothorax albumin, $g / L$ & $<20$ & Negative \\
\hline Acid-fast bacilli & Negative & Negative \\
\hline Lactate dehydrogenase, LDH, U/L & 50 & $120-250$ \\
\hline Carcinoembryonic antigen, CEA, ng/mL & 0.75 & $<4.7$ \\
\hline Neuron-specific enolase, NSE, ng/mL & 1.71 & $<16.3$ \\
\hline
\end{tabular}

TC, total cholesterol; TG, triglyceride; $\mathrm{LDH}$, lactate dehydrogenase; CEA, carcino-embryonic antigen; NSE, neuron-specific enolase.

The true chylous fluid contains chylous particles, shows a positive chylous test with high triglyceride levels in the pleural effusion and total cholesterol to triglyceride ratio of $<1$ (7). Causes of true chylothorax are either a thoracic duct injury or a lesion. In the present patient with primary nephrotic syndrome, the pleural effusion was milky white, and the chylous test was positive, both consistent with a true chylothorax (8). If the diagnosis is uncertain, when the pleural effusion is not chylous, or when other diseases cannot be ruled out, surgical exploration can be a method of diagnosis.

There are many reasons for the formation of a chylothorax. However, the three main mechanisms, according to Valentine et al., are: (I) leakage from the thoracic duct or its tributaries, (II) extravasation from pleural lymphatics, and (III) transdiaphragmatic flow of chylous ascites (9). Based on this, increased lymphatic vessel pressure or a change of permeability likely leads to poor reflux of the chylous fluid and induces chylothorax.

The mechanism of primary nephrotic syndrome, complicated with chylothorax, is unclear. In this case, we considered that the hypoproteinemia changed the permeability of the mucous membranes and lymphatic vessels, leading to chylous particles' leakage and chylous pleural effusion formation. Chylothorax may also have been caused by severe tissue edema, edema of the lymphatic vessels, and increased pressure, resulting in increased permeability or rupture of the walls of the lymphatics, and leakage of chylous fluid into the thoracic cavity.

Riley et al. considered that increased hydrodynamic pressure in the lymphatics causes back pressure with dilatation of the lymph vessels, retrograde flow, collateralization, and diffusion of the contents through the vessel walls into the serous spaces or lumina, parenchyma, or surfaces of organs (10). If the back pressure is severe enough or there is a structural disease of the lymphatics, rupture can occur with free leakage (11).

Treatment of the underlying cause of chylothorax is essential, but the daily loss of chylous fluid and resulting metabolic disorder also play critical roles in treatment. For this patient, we administered anticoagulant because nephrotic syndrome can easily cause a hypercoagulable state, and lipid-lowering drugs, as well as advise a lowfat diet, reducing foods containing too much cholesterol, increased physical activity, reduced weight, cessation or moderation of smoking and drinking, and regular selfadministration of the medications (e.g., atorvastatin, etc.). Diuresis and supplementary albumin symptomatic treatment can reduce tissue edema, pleural effusion, and chylothorax. The patient's pleural effusion was significantly reduced after active treatment, and the symptom of chest tightness was relieved.

Nephrotic syndrome is common in patients with chronic kidney disease. With improvements in socioeconomic status, lifestyle, and living environment, complications have also increased significantly. Although acute kidney injury, thromboembolic events, and infections are the major complications, some rare complications are now gaining more prominence. Therefore, it is crucial to understand the complications of nephrotic syndrome for precise diagnosis, treatment, and improvement of its clinical control.

Chylothorax is a rare complication encountered in primary nephrotic syndrome. It can be misdiagnosed if clinicians are unaware of it through a lack of relevant diagnostic and treatment experience. By reporting this case, we hope to raise awareness among physicians of the importance of pursuing an early diagnosis and identifying complications, which will improve clinicians' understanding of MN complications in primary nephrotic syndrome, enrich clinical knowledge, and provide suitable treatment options for future clinical reference. 


\section{Acknowledgments}

We thank the patient's family for their cooperation.

Funding: This work was supported by the Shanghai Shenkang Hospital Development Center (No. SHDC12016109).

\section{Footnote}

Reporting Checklist: The authors have completed the CARE reporting checklist. Available at https://apm.amegroups. com/article/view/10.21037/apm-21-1872/rc

Peer Review File: Available at https://apm.amegroups.com/ article/view/10.21037/apm-21-1872/prf

Conflicts of Interest: All authors have completed the ICMJE uniform disclosure form (available at https://apm. amegroups.com/article/view/10.21037/apm-21-1872/coif). The authors have no conflicts of interest to declare.

Ethical Statement: The authors are accountable for all aspects of the work in ensuring that questions related to the accuracy or integrity of any part of the work are appropriately investigated and resolved. All procedures performed in this study were in accordance with the ethical standards of the institutional and/or national research committee(s) and with the Helsinki Declaration (as revised in 2013). Written informed consent was obtained from the patient for publication of this case report and accompanying images. A copy of the written consent is available for review by the editorial office of this journal.

Open Access Statement: This is an Open Access article distributed in accordance with the Creative Commons Attribution-NonCommercial-NoDerivs 4.0 International License (CC BY-NC-ND 4.0), which permits the noncommercial replication and distribution of the article with the strict proviso that no changes or edits are made and the original work is properly cited (including links to both the

Cite this article as: $\mathrm{Xu} \mathrm{Y,} \mathrm{Shi} \mathrm{J,} \mathrm{Xu} \mathrm{S,} \mathrm{Cui} \mathrm{C.} \mathrm{Primary}$ nephrotic syndrome complicated by chylothorax: a case report. Ann Palliat Med 2022;11(7):2523-2528. doi: 10.21037/apm-21-1872 formal publication through the relevant DOI and the license). See: https://creativecommons.org/licenses/by-nc-nd/4.0/.

\section{References}

1. Bewick DJ, Johnstone DE, Landrigan PL. Primary chylopericardium associated with allergic alveolitis. Can Med Assoc J 1984;130:1577-9.

2. Poswal L, Dhyani A, Malik P, et al. Bilateral Chylothorax due to Brachiocephalic Vein Thrombosis in Relapsing Nephrotic Syndrome. Indian J Pediatr 2015;82:1181-2.

3. Tutor JD. Chylothorax in infants and children. Pediatrics 2014;133:722-33.

4. Weiss N, Solomon SB. Talc pleurodesis mimics pleural metastases: differentiation with positron emission tomography/computed tomography. Clin Nucl Med 2003;28:811-4.

5. Doerr CH, Miller DL, Ryu JH. Chylothorax. Semin Respir Crit Care Med 2001;22:617-26.

6. Noriega Aldave AP, Leslie JW. Transudative chylothorax in a patient with lymphangioleiomyomatosis. Respir Med Case Rep 2017;22:271-3.

7. Thaler MA, Bietenbeck A, Schulz C, et al. Establishment of triglyceride cut-off values to detect chylous ascites and pleural effusions. Clin Biochem 2017;50:134-8.

8. Lin WY, Lin GM, Wu CC. Coexistence of noncommunicated chylothorax and chylous ascites in nephrotic syndrome. Nephrology (Carlton) 2009;14:700.

9. Valentine VG, Raffin TA. The management of chylothorax. Chest 1992;102:586-91.

10. Riley LE, Ataya A. Clinical approach and review of causes of a chylothorax. Respir Med 2019;157:7-13.

11. Pui MH, Yueh TC. Lymphoscintigraphy in chyluria, chyloperitoneum and chylothorax. J Nucl Med 1998;39:1292-6.

(English Language Editors: K. Brown and J. Chapnick) 